\title{
El papel de la mujer en la explotación de ganado bovino de doble propósito en Ecuador
}

\author{
Torres, Y. ${ }^{1} ;$ Pablos-Heredero, C. de ${ }^{2}$; Morantes, M. ${ }^{3}$; Rangel, J. ${ }^{4}$; Espinosa, J.A. ${ }^{4}$; Angón, E. ${ }^{5}$ y García, A. ${ }^{5}$
}

\author{
'Universidad Técnica Estatal de Quevedo. Quevedo. Ecuador. \\ 2Departamento de Economía de la Empresa, Economía Aplicada II y Fundamentos de Análisis Económico. Universidad Rey Juan Carlos. \\ Madrid. España. \\ ${ }^{3}$ Departamento de Producción Animal. Universidad Central. Venezuela. \\ ${ }^{4}$ INIFAP. México. \\ ${ }^{5}$ Departamento de Producción Animal. Universidad de Córdoba. España.
}

\section{PALABRAS CLAVE ADICIONALES}

Sistemas de subsistencia.

Explotación familiar.

\section{RESUMEN}

\begin{abstract}
Uno de los objetivos estratégicos para FAO es disminuir la brecha de género en aras del desarrollo rural. El presente trabajo se planteó para conocer la percepción que tienen los productores del sistema de doble propósito ecuatoriano sobre las diferencias de género, tanto en el ámbito familiar como en la unidad de producción. Se tomó una muestra aleatoria de 132 unidades familiares-productivas del bosque tropical de Manabí. La información fue obtenida a través de una encuesta cara a cara, donde se preguntaron cuestiones relacionadas con el perfil socioeconómico de la familia, el sistema de producción y la percepción del papel de los distintos géneros. Los resultados muestran que existen importantes diferencias de género en el mundo rural ecuatoriano. Los productores tienen fuertes creencias en las diferencias sociales y biológicas entre sexos. El trabajo y rol de mujer es percibido únicamente relevante en el ámbito doméstico. Mientras que en la unidad productiva apenas asume responsabilidades. La toma de decisiones es exclusiva del hombre cuando se trata del ámbito empresarial; mientras que la mujer participa junto a él en decisiones del hogar, aunque nunca asume un papel predominante.
\end{abstract}

\section{The woman role in the Ecuatorian bovine double purpose systems}

\section{SUMMARY}

One of the strategic objectives for FAO is to reduce the gender gap in the interests of rural development. This paper was raised to know the perception that the producers of the Ecuadorian double purpose bovine have on gender differences both in the family and in the production unit. A random sample of 132 family-productive units of tropical forest from Manabi was used. The information was obtained through a survey related to socioeconomic profile of the family, the production system and the perception of the role of the different genders. Results show that there are significant gender differences in rural Ecuador. Producers have strong beliefs in social and biological differences between sexes. The work and role of women is perceived only relevant in the domestic sphere. While in the production unit just assume responsibilities. Decision making is unique to man when it comes to the business world; while women participate with him in household decisions.

\section{INFORMACIÓN}

Cronología del artículo.

Recibido/received: 12.05 .15

Aceptado/Accept: 08.04.16

Online: 15.09 .16

Correspondencia a los autores/Contact e-mail:

guisell2010@hotmail.com

\section{INTRODUCCIÓN}

La disminución de la brecha de género en aras del desarrollo rural es un objetivo estratégico para la FAO (2011). El Informe sobre Producción y Salud Animal (FAO,
2012) define a las mujeres rurales como las guardianas invisibles de la diversidad ganadera. Pobreza, seguridad alimentaria, desnutrición, biodiversidad y conservación son atributos estrechamente ligados a los países en vía de desarrollo. Frecuentemente, el papel de la mujer en 
el ámbito rural de los países en vías de desarrollo es poco visible, aunque resulta clave en la administración de hogares generalmente complejos. Por ejemplo, en Somalia, se han puesto de manifiesto estrategias de subsistencia llevadas a cabo por mujeres, donde la comercialización de leche de camella es el eje de la generación de valor (FAO, 2011).

La situación actual de las mujeres rurales en Ecuador es una preocupación constante, tal y como se recogen en los lineamientos estratégicos del Plan Nacional del Buen Vivir para el periodo 2013-2017. De acuerdo con Ballara et al. (2012), el principal problema es la exclusión; las mujeres suelen permanecer en el sector informal de la economía, no acceden proporcionalmente a la propiedad de los factores de producción ni participan en la toma de decisiones. Además el trabajo de la mujer es poco visible y en gran medida suele ser subestimado (FAO, 2008).

El reconocimiento del trabajo de la mujer es el primer paso para afrontar la exclusión; aunque en muchas ocasiones, no existe una percepción nítida del papel que desempeñan en el ámbito rural. Además existe una gran brecha cultural y educacional en el ámbito rural frente al urbano. Los bajos niveles socioculturales predominantes en un entorno muy tradicional y dominado por el género masculino son factores que apuntan hacia una escasa valoración del trabajo de la mujer rural ecuatoriana.

Por otra parte, los sistemas de producción predominantes en el ámbito rural ecuatoriano son típicamente familiares de subsistencia, por lo que la demarcación entre actividad agropecuaria y ámbito familiar es prácticamente inexistente. En consecuencia, el trabajo de la mujer en los diferentes ámbitos, probablemente, sea menos visible que en otros entornos donde las tareas están mejor definidas y los ámbitos naturalmente delimitados.

Uno de los sistemas de subsistencia más representativos es la explotación de ganado bovino de doble propósito (Torres et al. 2014). Se localiza mayoritariamente en los bosques tropicales de la Costa, donde predominan bajos niveles socioculturales y el riesgo de marginalidad es elevado (Díaz et al., 2011; Van't Hooft y Wollen, 2012). El sistema sigue un modelo familiar y se integra por pequeños productores orientados a la subsistencia de la familia mediante una estrategia de mínimo coste con baja eficiencia (Hernández et al., 2013) y

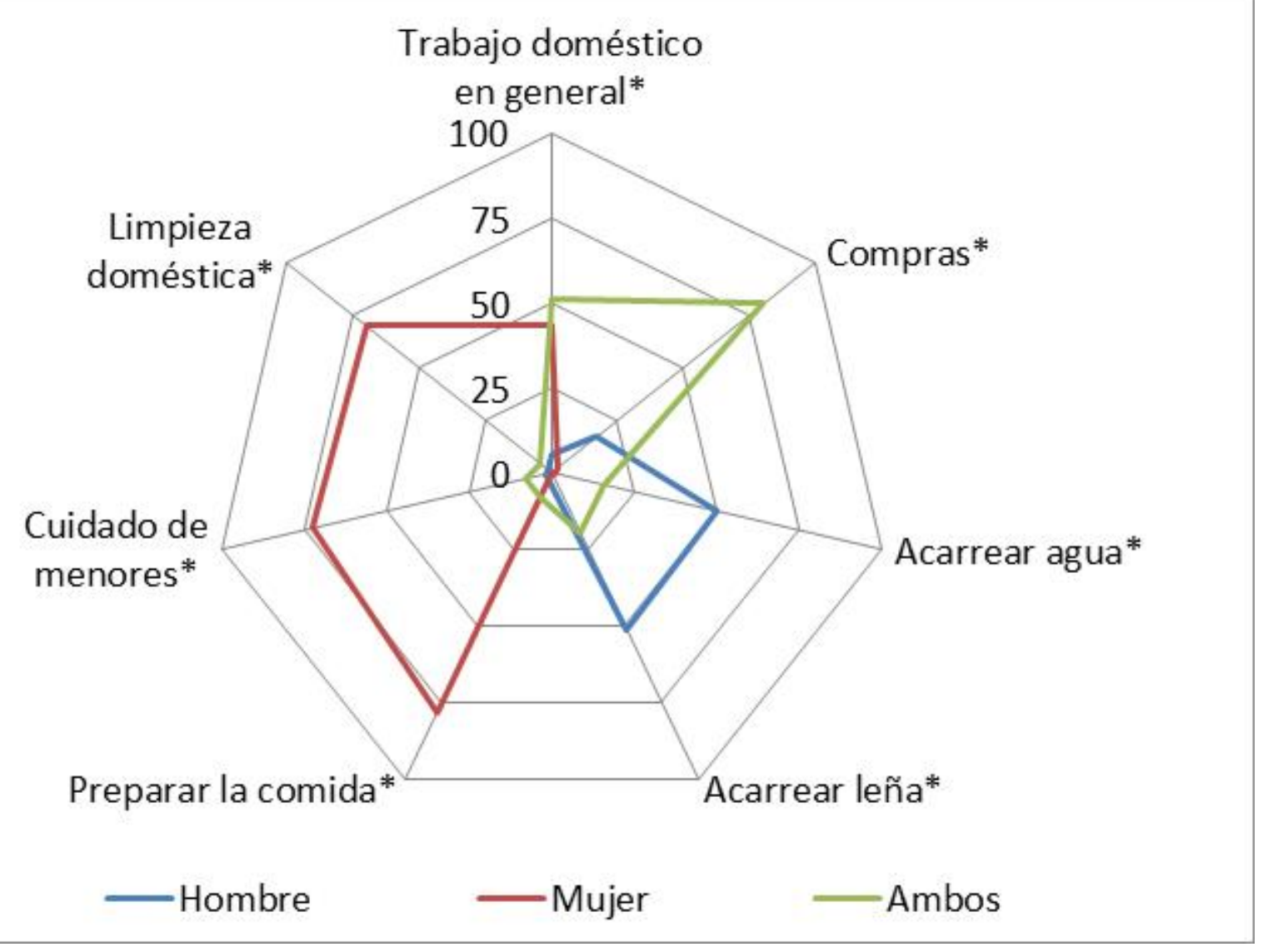

Figura 1. Diferencias de género en actividades de ámbito familiar (las variables marcadas con * son significativamente diferentes; $p<0,05)$ (Gender differences in home activities (variables with * are significantly different; $p<0.05$ ). 

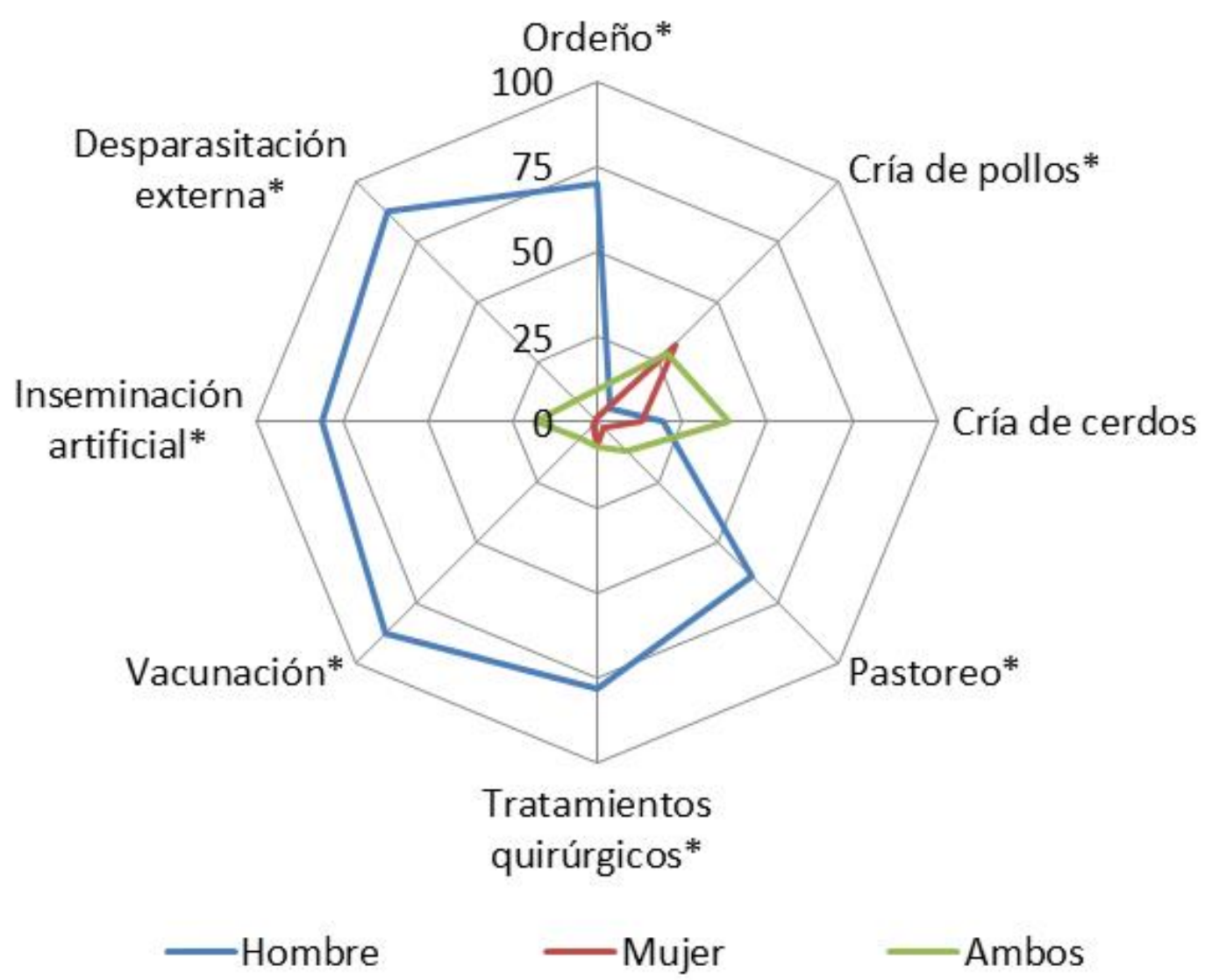

-Ambos

Figura 2. Diferencias de género en actividades ganaderas (las variables marcadas con * son significativamente diferentes; $p<0,05)$ (Gender differences in livestock activities (variables with * are significantly different; $p<0.05$ ).

escaso nivel tecnológico, que viven en el umbral de la pobreza. Las explotaciones siguen esquemas de producción tradicionales y están limitadas por la exclusión de los circuitos formales de crédito, la dificultad de acceso a insumos externos y su bajo nivel tecnológico (Albarrán et al., 2015; Rangel et al., 2015).

Por tanto, se plantea como objetivo conocer la percepción que tienen los productores del sistema de doble propósito sobre las diferencias de género, tanto en el ámbito familiar como en la unidad de producción.

\section{MATERIAL Y MÉTODOS}

El área de estudio fue la provincia de Manabí, que concentra el 9,41 \% de la producción lechera nacional y se sitúa en el bosque tropical de la región Costa (Haro, 2003). La unidad familiar y de producción tipo tiene una dimensión media de 16 vacas, con un coeficiente de variación del 104 \%. El tamaño del hato es de 41 cabezas totales (incluyendo reproductoras, sementales y reposición) de raza criolla y animales híbridos entre Bos taurus $\mathrm{x}$ Bos indicus y la superficie media de 44,32 ha, con una carga ganadera de 2,89 UGM/ha. Una descripción más detallada del sistema DP se encuentra en Torres et al. (2014).

Se tomó una muestra aleatoria de 132 unidades familiares-productivas del bosque tropical de Manabí. La información fue obtenida a través de una encuesta cara a cara, donde se preguntaron cuestiones relacionadas con el perfil socioeconómico de la familia, el sistema de producción y la percepción del papel de los distintos géneros en la realización del trabajo doméstico, agricultura, ganadería, gestión y toma de decisiones. El 80 \% de los encuestados fueron hombres; en el $20 \%$ restante también participaron mujeres junto al hombre.

Se obtuvieron las frecuencias de cada variable y la percepción de género fue analizada mediante test de bondad de ajuste.

\section{RESULTADOS Y DISCUSIÓN}

El $95 \%$ de las explotaciones son propiedad de la familia, y casi el $55 \%$ de los productores son analfabetos o cuentan con educación primaria. El 60,2\% delimitó ambos géneros por diferencias biológicas; destacando un 


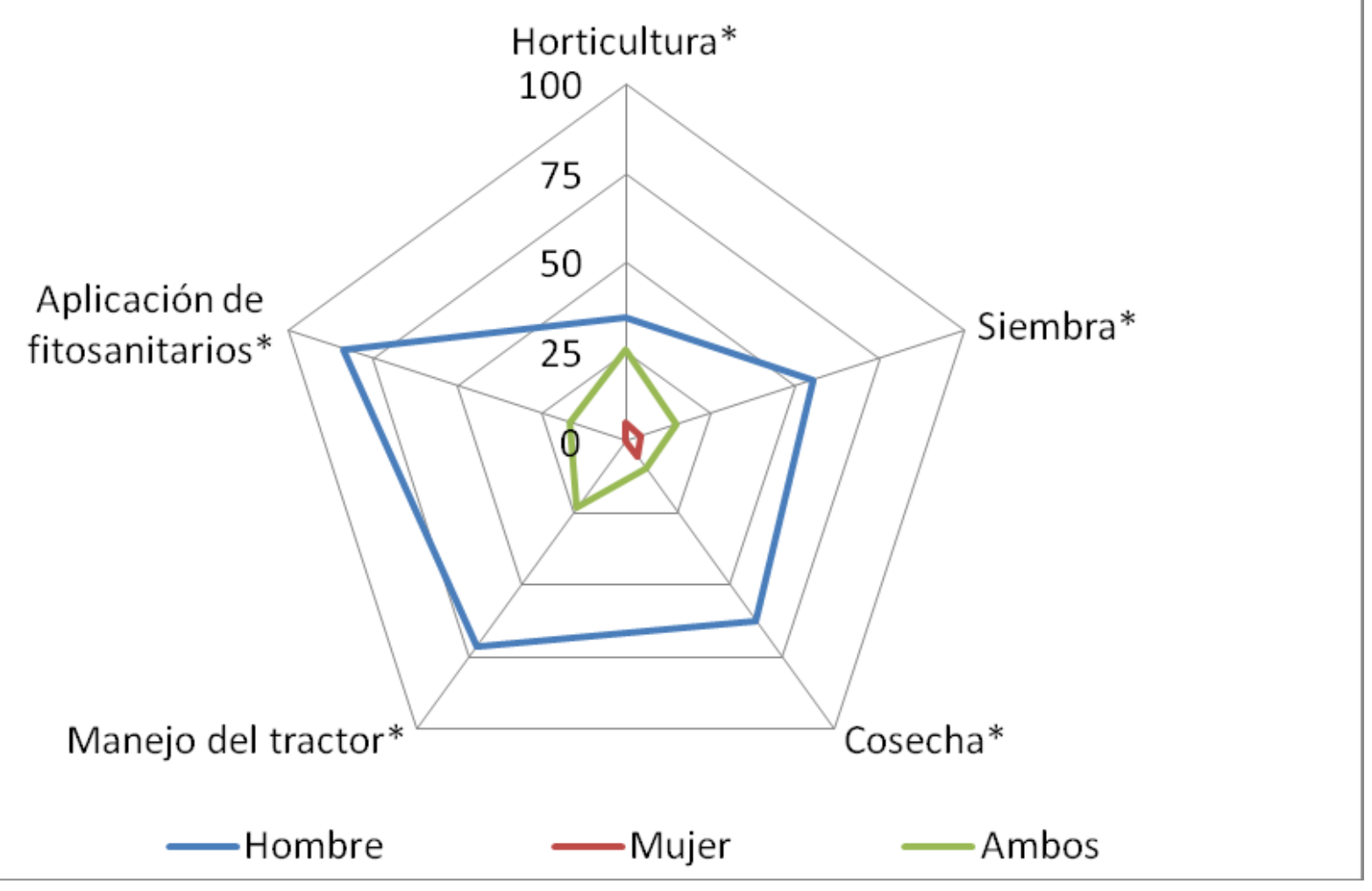

Figura 3. Diferencias de género en actividades agrícolas (las variables marcadas con * son significativamente diferentes; $\mathrm{p}<0,05)$ (Gender differences in agricultural activities (variables with * are significantly different; $p<0.05$ ).

$39,8 \%$ que consideró también diferencias sociales. Es notable que exista un $16 \%$ de familias donde la mujer no come a la vez que el resto de la familia; o bien el hombre come primero o bien la mujer come la última. Aunque casi el 100\% consideró que la escolarización es igual de importante en niños y en niñas.

Los productores tienen la percepción de que sus mujeres trabajan más que ellos; sin embargo, el 98,5\% consideró que no es importante la contribución de la mujer en la actividad agropecuaria.

En la figura 1 se indican las diferencias de género percibidas por los productores en el ámbito doméstico. Los roles están claramente definidos y es la mujer quien desarrolla mayoritariamente las actividades relacionadas con el hogar. Sin embargo, la percepción predominante general es que las tareas domésticas son compartidas. Esta percepción cambia cuando se evalúan las tareas individualmente. Las tareas domésticas complejas que exigen responsabilidad son desarrolladas casi exclusivamente por mujeres: preparar la comida, cuidar los niños y la limpieza del hogar. En las compras domésticas es donde el hombre lleva el rol predominante, bien sólo o bien acompañado de su mujer; contraviniendo la tendencia general. Si la mujer se dedica al hogar, es ella quien mejor sabe las necesidades de compra y, por tanto, quien debería asumir la responsabilidad. Esto apunta a que las cuestiones monetarias, sea cual sea el ámbito, son dominadas por el hombre. Por otra parte, hay actividades como acarreo de agua y leña que son consideradas masculinas y la mujer no participa. Estas actividades son sencillas, rutinarias y de baja responsabilidad.

Las actividades ganaderas son ámbito casi exclusivamente masculino (figura 2). La mujer circunscribe su papel a producciones de traspatio, fundamentalmente en la crianza de aves y cerdos. En el ganado bovino, actividad principal de la explotación, la percepción de los productores es que participa muy discretamente.

En las actividades agrícolas es donde la mujer, según los propietarios, menos participa (figura 3). La aplicación de fitosanitarios y las actividades que implican el uso de maquinaria agrícola son tareas netamente masculinas. Sólo en la producción de hortalizas el hombre comparte tareas con otros miembros de la familia; aunque apenas participa la mujer.

En la figura 4 se indican las diferencias de género percibidas por los productores en actividades de gestión. De nuevo es el hombre quien desempeña casi exclusivamente todas las tareas. Donde menos interviene la mujer es en las relaciones técnicas de la explotación. Adquiere cierta relevancia en las relaciones con la administración. 


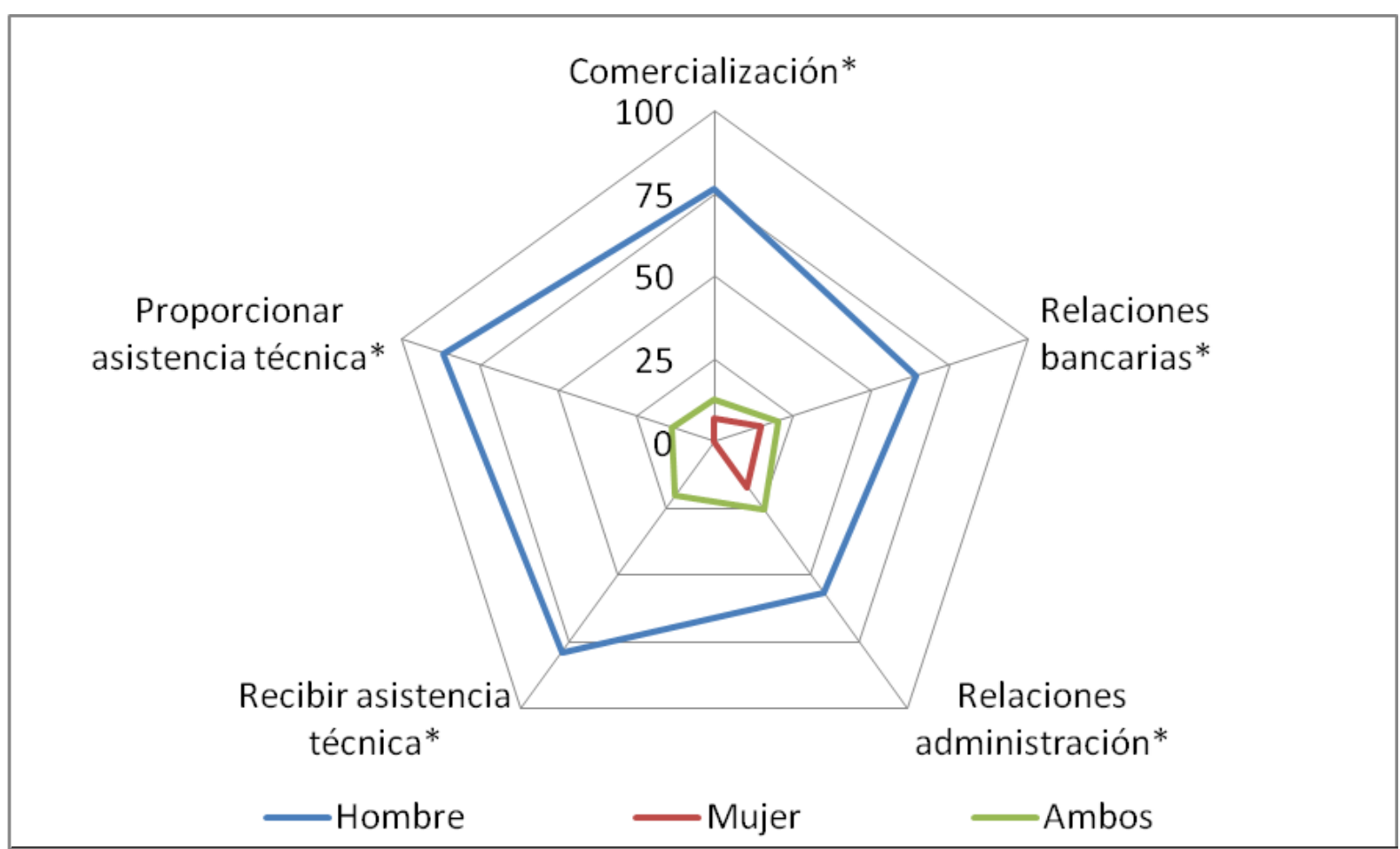

Figura 4. Diferencias de género en actividades de gestión (las variables marcadas con * son significativamente diferentes; $p<0,05)$ (Gender differences in managerial activities (variables with * are significantly different; $p<0.05$ ).

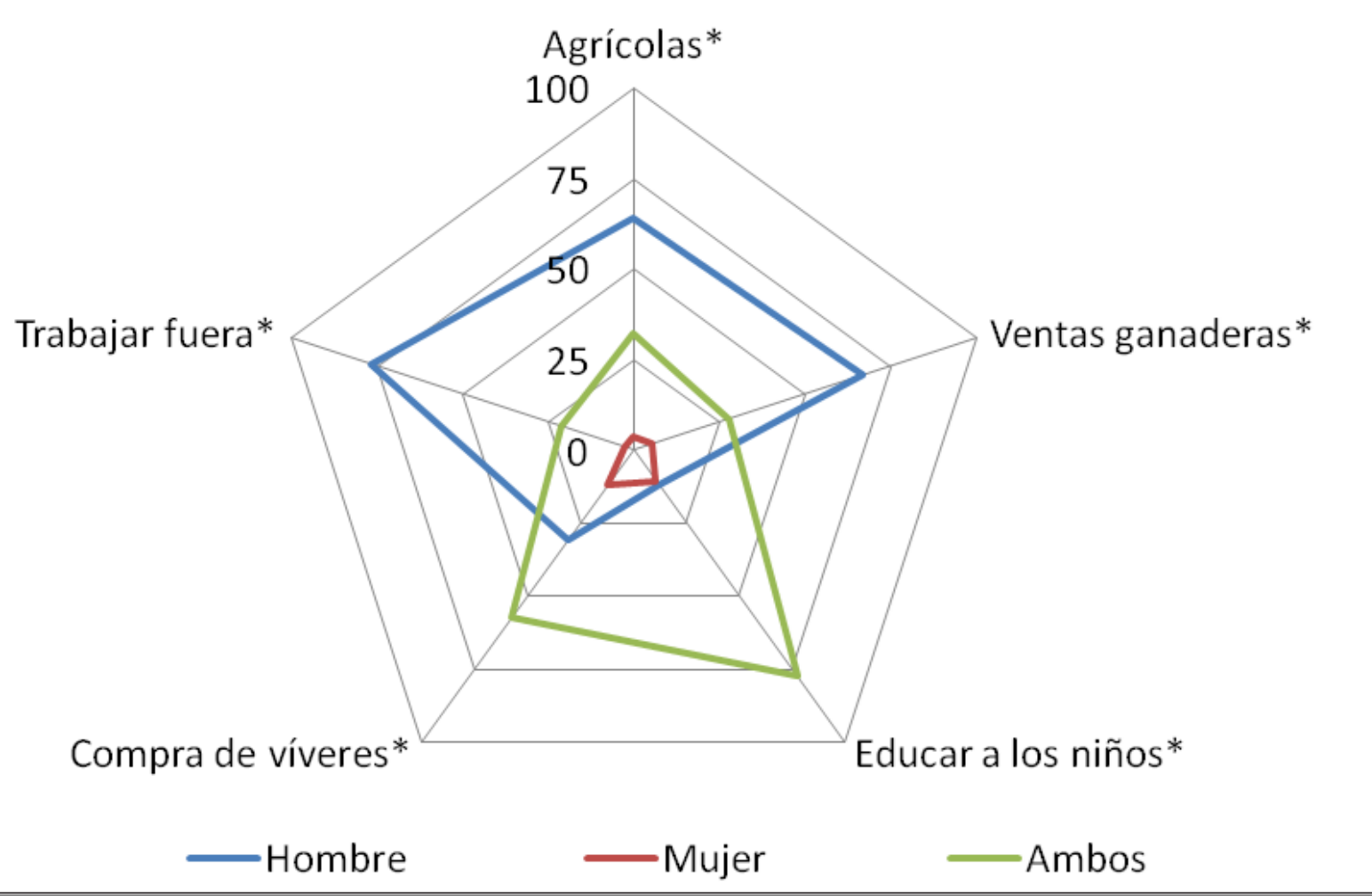

Figura 5. Diferencias de género en la toma de decisiones (las variables marcadas con * son significativamente diferentes; $p<0,05)$ (Gender differences in decision making process (variables with * are significantly different; $p<0.05$ ). 
La toma de decisiones está dominada por los hombres y sólo en la compra de víveres y en la educación de los hijos la mujer comparte responsabilidad con el hombre (figura 5). Las decisiones agrícolas, comerciales y laborales son adoptadas generalmente por los hombres.

\section{CONCLUSIONES}

Existen importantes diferencias de género en el ámbito rural ecuatoriano. Los productores tienen fuertes creencias en las diferencias sociales y biológicas entre sexos. En consecuencia, el trabajo y rol de mujer es percibido únicamente relevante en el entorno doméstico, mientras que en la unidad productiva apenas asume responsabilidades. La toma de decisiones es exclusiva del hombre cuando se trata de aspectos empresariales; mientras que la mujer participa junto a él en decisiones del hogar, aunque nunca asume un papel predominante.

\section{AGRADECIMIENTOS}

El estudio se ha desarrollado dentro del Proyecto Caracterización técnica y económica del sistema bovino de doble propósito en la Provincia de Manabí(Ecuador). Mejora del nivel de competitividad financiado por el FOCICYT de la Universidad Técnica Estatal de Quevedo (Ecuador).

\section{BIBLIOGRAFÍA}

Albarrán, P.B.; Rebollar, R.S.; García, M.A.; Rojo, R.R.; Avilés, N.F. and Arriaga, J.C. 2015. Socioeconomic and productive characterization of dual purpose farm oriented to milk production in a subtropical region of Mexico. Trop Anim Health Produc, 47: 519-523.

Ballara, M.; Damianoviæ, N. y Valenzuela, R. 2012. Mujer, agri- cultura y seguridad alimentaria: una mirada para el fortalecimiento de las políticas públicas en América Latina. Thematic Consultation. $15 \mathrm{pp}$.

Díaz, P.; Oros, V.; Vilaboa, J.; Martínez, J.P. y Torres, G. 2011. Dinámica del desarrollo de la ganadería doble propósito en las Choapas, Veracruz, México. Trop Su Agro, 14: 191-199.

FAO. 2008. Situación de las mujeres rurales. Ecuador. Organización de las Naciones Unidas para la Agricultura y la Alimentación. Roma. Italia. 156 pp.

FAO. 2011 . Estado mundial de la agricultura y la alimentación. 2010-2011. Las mujeres en la agricultura. Cerrar la brecha de género en aras del desarrollo. Organización de las Naciones Unidas para la Agricultura y la Alimentación. Roma. Italia.

FAO. 2012. Invisibles guardians. Women manage livestock diversity. Animal Production and Health Paper. Roma. Italia. 174 pp.

Haro, R. 2003. Informe sobre recursos zoogenéticos Ecuador. Ministerio de agricultura y ganadería y subsecretaria de fomento agroproductivo. Dirección para la implementación del desarrollo agropecuario, agroforestal y agroindustrial. Quito. Ecuador. 21 pp.

Hernández, P.; Estrada, J.G.; Avilés, F.; Yong, G.; López, F.; Solís, A.D. y Castelán, O.A. 2013. Tipificación de los sistemas campesinos de producción de leche del sur del estado de México. Universidad y Ciencia. Trópico Húmedo UJAT, 29: 1931.

Rangel J.; Torres Y.; De Pablos-Heredero, C. and Espinoza J.A. 2015. Identification of technological areas for dual purpose cattle in Mexico and Ecuador. 66 $6^{\text {th }}$ Annual Meeting of the European Federation of Animal Science EAAP. 31 August-4 September. Warsaw. Poland.

Torres Y.; Rivas J.; De Pablos Heredero C.; Perea J.; Toro Mújica P.; Angón, E. y García, A. 2014. Identificación e implementación de paquetes tecnológicos en ganadería vacuna de doble propósito. Caso Manabí-Ecuador. Rev Mex Cien Pec, 5: 393-407.

Van't-Hooft, K. and Wollen, T. 2012. Sustainable livestock management for poverty alleviation and food security. Cab international. London. UK. 194 pp. 